\title{
Role of the Social Capital in Effectiveness of Knowledge Management within Iran Khodro Diesel Company
}

\author{
Ali Akbar Ahmadi \\ Public management Department, Payame Noor University, 19395-3697 Tehran, I.R. of IRAN
}

Accepted: December 12, 2011 Published: January 9, 2012

Doi:10.5296/ijld.v2i1.1255 URL: http://dx.doi.org/10.5296/ijld.v2i1.1255

\begin{abstract}
:
Social capital is considered as a new concept which, as compared with the human or physical capitals, is of more significance within different organizations. On the other hand the modern management has already identified the concept of knowledge as an endless source of interests and competitive advantages. Given the growing significance of this organizational necessity which is vital for proper knowledge management all the required efforts shall be made for identifying and understanding the factors affecting and the factors affected by the concept of knowledge.

The main purpose behind the present research was to study role of the social capital and its relationship with the knowledge management effectiveness within every organization. The statistical community used for the present research included staff of the Iran Khodro Diesel Company Research and Development Department. The researcher, at the beginning of his research, referred to three secondary assumptions and one primary assumption. Then the social capital was designed based on three dimensions including cognitive, relational and structural dimensions and the knowledge management was designed based on three dimensions of the knowledge, knowledge storage, continuous knowledge refinement, knowledge utilization and knowledge sharing/distribution. The main tools for collecting the required data included statistical sampling for 105 individuals as well as the social capital and knowledge management related questionnaires distributed among the participants in the research. The validity of the questionnaires was verified by use of the content validity method and the factor analysis of the structural equations. Also the reliability of the questionnaires was calculated based on the Cronbach's Alpha at 0.89 and 0.91 which was indicative of an accepted reliability. The statistical analysis was made by use of the SPSS statistical and LISREL software. Accordingly, all the three secondary assumptions as well as the primary assumption were confirmed. Based on the outcome of the research it was verified that there is
\end{abstract}


a meaningful relationship between the three dimensions of the social capital and the knowledge management effectiveness. In continuation the organizational rating was assessed based on the knowledge management dimensions and the social capital and in the end some recommendations were introduced for promotion of the social capital and knowledge management within the organization.

\section{Introduction:}

Recent progress transpired in the information technology has remarkably lowered the data management costs. Such developments have led to insertion of new concepts into the management literature such as learning organization, knowledge organization and knowledge management. Today all organizations are attempting to promote innovation in their activities, processes, products and services with the purpose of improving their competitiveness by utilizing knowledge management strategies. It is worth mentioning that in the today dynamic, challenging and competitive business climate all organizations, for their success, are required to be turned into leaning organizations. Accordingly all organizations shall be able to acquire the knowledge needed for creation of innovation in their products and improvement of their processing activities distributing it among their staff and utilizing it in all their daily and routine activities. This is the only way for these organizations to be able to respond to the requirements of the competitive business climate and meet the rapidly changing demands of their customers. Identification of factors affecting the organizational knowledge management is considered as among the first measures which shall be adopted in effective use of the intellectual capital within every organization. On the other hand the social capital is one of the new concepts which is playing more important role in every organization or society as compared with the human and physical capitals. Today, in the fields of sociology and economy and recently the organizational management concept of the social capital is widely utilized. The concept of the social capital in fact refers to the bindings and relationships between members of a network as a valuable source which by creating norms and mutual trust will prepare the ground for achievements of all objectives desired by the members of every organization. In absence of the social capital other capitals will lose their effectiveness and the march towards development and cultural and economic evolution becomes more difficult and risky. The social capital, can give us a new impression of the socio-economic systems assisting the managers in better management of their systems and organizations whether at macro management level or within small organizations and corporations. The present research intended to study role of the social capital in the effectiveness of the knowledge management. Given the definitions and dimensions of the knowledge management and the social capital which lay emphasis on the relationships and interactions among individuals the researcher reached the conclusion that the relationship between the social capital and the effectiveness of the knowledge management could be investigated and extent of the role of the social capital in the effectiveness of the knowledge management could be tested and assessed accordingly.

The main purpose question facing the present research was how we could manage the knowledge within every organization in order to produce its highest rank of effectiveness? 
Due to the significance of effectiveness of the knowledge management and the social capital and the studies already carried out in this respect the researcher attempted to test the relationship between the knowledge management effectiveness and the social capital. Major questions raised in the present research was whether principally there exists a relationship between the social capital and the knowledge management or not; and whether high level of the social capital in any organization will lead to further effectiveness of the knowledge management or vice versa or not?

\section{Literature Research:}

Some experts consider the social capital as a collection of different elements of the social organization including norms, social networks, and mutual trust which may facilitate cooperation and mutually supportive relations among individuals for the purpose of their common interests (Putnam, 1995).

From the organizational point of view Nahapiet and Ghoshal (1998) have defined the social capital as sum of actual and potential resources embedded within available through and derived from the network of relationships possessed by an individual or social unit. They (Nahapiet and Ghoshal, 1998) also think that the social capital is one of the most important organizational assets and qualifications which can extraordinarily assist every organization in knowledge creation and sharing/distribution and lead the organization to sustainable organizational advantages as compared with other organizations. As Cohen and Prusak (2001) opine, the idea for attending the social capital within organizations is relatively a new thought. As Adler and Kwon (2002) believe, given the childhood period and application of the social capital in the organizational life all the studies so far carried out are indicative of the fact that the social capital can support tacit knowledge of every individual. Within the same respect Nahapiet and Ghoshal (1998), holding an organizational approach, are dividing different aspects of the social capital in three categories of cognitive, relational and structural dimensions as follows

Cognitive dimension: The cognitive dimension of the social capital refers to the resources which provide features, comments and meaning systems shared among different groups (Icourel, 1973).

Common language and codes: Because of various reasons common language affects knowledge composition and knowledge exchange conditions. The first reason goes to the fact that language is of direct and important application in the social relations; language is a tool that facilitates exchange of views among individuals and eases exchange of information among them. By use of common language people ask questions from each other and carry out their trade activities within the society. The second reason goes to the fact that language affects our understandings and the relevant codes provide us with a reference in favor of our reading and understanding from our surroundings. And the third reason; is that common language promotes the ability to combine pieces of information.

Common anecdotes: In addition to common language and codes some researchers believe that myths, anecdotes and metaphors provide powerful tools and means within societies for creating, exchanging and keeping rich collections of meanings. 
Orr (1990) proved how anecdotes would facilitate exchange of experiences among experts. Therefore emergence of common anecdotes within a society will lead to creation and transferring new comments on various events and give rise to creation of composition of different forms of knowledge which generally are hidden.

Structural dimension: The structural dimension refers to the relationships among actors who are consecutively are sharing information. Nahapiet and Ghoshal (1998) inferred that access to such information would promote capabilities of every organization in knowledge absorption (analysis) and knowledge integration creating a competitive advantage for the organization (Carrie R. Leana and Frits K. Pil, 2006).

The structural dimension of the social capital includes bindings between the network and composition of the network organization and organizational fit (Nahapiet and Ghoshal, 1998: 251).

The structural dimension of the social capital is derived from the structural form, diversity, centrality and supportive role of the participants (Krosi, 2007: 531).

Relational dimension: The relational dimension includes nature of the relationships within an organization. In other words while the structural dimension is speaking of the connection between the staff within an organization the relational dimension is speaking of the essence and quality of such relationship (for example whether such relationships are defined by trust, sincerity, passion and... or not?)(Bolino, 2002:506). This dimension includes trust, norms, obligations and identification (Nahapiet and Ghoshal, 1998: 251).

Relational dimension refers to the personal relations among individuals; the relations which may be developed by interaction that is the extent of trust, obligation and mutual relations among different groups (Krossi, 2007: 531).

Nahapiet and Ghoshal (1998) opine that any organization may provide institutionalized environment which is conducive for development of the social capital. They believe that composition and exchange of knowledge can be further facilitated when individuals are further connected with each other (structural capital); when individuals enjoy positive and strong relationships with each other (relational capital); and when individuals are capable of understanding and utilizing knowledge (cognitive capital) (Mat Isa and Ameer, 2007: 248).

Peter Drucker in his book titled "Post-Capitalist Society" says that the primary economic resource is no longer capital, land, or labor; now the primary economic resource is the knowledge which is and will always be considered as the fundamental asset of every successful economy (Radding, 2004) and it will outstandingly supersedes all physical and financial assets; in all systems knowledge and knowledge creation are considered as the vital element for survival of every organization within competitive environments (Ebillie et.al) therefore in the present era which is called as the era of knowledge every organization is needed to use knowledge in different ways in order to achieve sustainable competitive advantages taking fruitful step for optimum use of the resources of the organization (Martenson, 2001).

Knowledge management includes identification, utilization, organization and processing of the available information for the creation of knowledge which shall subsequently be shared with others towards further creation of knowledge (Radding, 2004). Knowledge management is in fact a kind of technology which is knowledge-oriented and it intends to 
settle all the existing problems by effective use of knowledge (J. Michael and Geraldas, 2004). Given all the above-mentioned we can produce the following definition for the knowledge management:

"Knowledge management is the process of creating, collecting, sharing/ distributing, continuous refining and utilizing the knowledge. By establishment and use of new systems including knowledge management even if we cannot achieve all the desired goals for promoting the staff efficiency and the organizational knowledge as well as updating the organizational knowledge, optimizing the production, increasing the capacity and capability for tackling all the organizational problems and tens of other desirable objectives but we can definitely resolve major parts of them. Establishment of the knowledge management system in every organization requires preparation of certain technical and scientific grounds. The technical ground includes computer systems and communication networks and the scientific ground includes tools for collecting and storing knowledge required by the organization. Meanwhile the first step is to study all the required grounds for establishment of the knowledge management system. Utilization of such a system and making progress in different scientific fields including the knowledge management are the preconditions for development and promotion of every society and organization. Accordingly establishment of the knowledge management system is considered as a valuable step towards further growth of every organization including the organization which is studied in the present research. Now in order for us to get further familiar with the subject we will discuss it in more detail as follows:

\subsection{Conversion of data into knowledge:}

One of the all time important issues in our discussion is the capacity for converting data into usable information that is knowledge. The Figure Number 1 specifies this important process. It is natural that all the available data cannot be directly converted into knowledge and firstly they shall be processed, refined and organized in order for them to be turned into information. Then as demonstrated in the Figure Number 1 the required knowledge is generated. It is noticeable the capacity of the knowledge generated is considered as the strong point in the process.

\subsection{Knowledge Collection:}

The first step towards creation and utilization of knowledge management is collection of knowledge which covers wide range of activities to this end including purchasing knowledge in the knowledge markets, recruiting those individuals who possess the required knowledge, promoting knowledge by holding scientific gatherings among the staff and many other relevant activities. Different methods for collecting and promoting knowledge within an organization are as follows:

a. Organizational rewards

b. Assessment and evaluation of the success of the programs on knowledge sharing/distribution 
c. Inclusion of the knowledge sharing/distribution activities as a precondition for employment of the staff

d. Promotion of knowledge sharing/distribution by use of internet

e. Promotion of sharing of the knowledge possessed by directors and managers

f. Establishment of new teams and groupings in relation to the subjects which are favored by the staff

g. Extending support to the experienced staff for the protection of knowledge and its transition to others (Watson, 2001:3)

Another mechanism for creating knowledge within an organization is the creation and development of the knowledge required by the organization through different means. Purchase of knowledge, development of research and scientific centers, effective communication with research centers out of the organization, continuous analysis of the rival organizations and purchase of knowledge-oriented organizations for acquiring their knowledge, all, are other activities which may be done for creating and developing knowledge.

\subsection{Knowledge storage and organization:}

As other important relevant issues we need to refer to the knowledge storage and organization. When lots of information and documents, texts, forms,... become available in favor of the organization their storage and organization become of great significance. Given the existing personal computers and local computer networks we may face huge amount of information which are needed to be managed and arranged accordingly. To this end all organizations may use assistance provided by the document management systems. For the same purpose codification of the organizational knowledge can be of valuable use. As one of the ways for organizing information, codification is converting organizational knowledge into a certain forms which can be used by all parties who require such knowledge. Codification converts knowledge into a set of frame works which will practically be usable by all and it is the organization which is responsible to identify cases wherein the knowledge shall be used. Since the main purpose of codification is to convert the knowledge into usable frameworks every organization shall consider and determine where and when the knowledge shall be used. This occurs while for this purpose the definition produced for knowledge and its application shall not remain confined. On the other hand deciding on the cases where the knowledge shall be used shall not be turned into a difficult task. For example Honda Company always registers and keeps records of its failed designs and projects because it believes that they may be usable and applicable in the future (Davenport, 1994). Those organizations which desire to use the codification method shall observe the following recommendations:

1) All directors and managers of the knowledge-based plans shall use the codification methods in favor of the trade activities of their organizations

2) The directors and managers shall not make mistake in identifying the knowledge which shall lead them to the objectives of the organization 
3) The directors and managers shall do the codification based on the usefulness and pertinence of the knowledge to the objectives of the organizations

4) Codification shall produce proper means for knowledge sharing/distribution ( Radding, 1994)

After storage and organization of knowledge its utilization and refinement shall be discussed and decided. Continuous and distribution of knowledge is one of the ways for promotion of knowledge among the staff. Preparation of the ground for constructive dialogue among staff is also fruitful for exchanging and distributing knowledge among them. Accordingly designing and putting into effect the system which can follow up regulated and continuous distribution of knowledge among the staff is one of the pressing requirements. It is notified that form of the knowledge sharing/distribution shall be in line with the culture of the organization and its desired objectives. On the other hand export and import of knowledge are interdependent and without import of knowledge its export will naturally be out of the question. It is also worth mentioning that possession of knowledge is of utmost importance but it cannot by itself ensure its practical use. Continuous information refinement as the continuous screening of information and knowledge available in the organization and its updating based on the latest requirements is considered as one of the pressing needs in the knowledge management process. Continuous knowledge refinement will promote the records within the organization making them more dynamic for acquiring, saving and organizing the required knowledge. After passing through the previous phases proper and effective use of knowledge can shed more light on the main intention of the knowledge management. Effective and proper utilization of knowledge actually depends on the knowledge management. It goes without saying that as long as the proper grounds for use of knowledge are not provided we cannot expect to witness effectiveness and efficacy of knowledge and enjoy its advantages.

Transfer of information and knowledge, at macro and micro levels, among individuals and organizations depends on the individuals who can start and facilitate the transfer. Therefore everything that impedes or facilitates relationships between individuals may affect knowledge transfer as. For the same reason significance of "trust" has always been emphasized in developing and applying knowledge. Trust becomes so important especially when the relations are of complex nature. Therefore role of trust in knowledge development is of paramount significance and by use of trust we can diminish the distrust and possible risks visible during knowledge transfer which by itself is necessary for effective performance of a knowledge-oriented economy.

In spite of the fact that "trust" is considered as a value commodity we cannot buy from any market. As Kennett Arrow points: "If you have to buy it, you already have some doubts about what you've bought". This occurs while establishment of trust depends upon a set of social values, cultural traditions and customs and common expectations. The behavioral models established based on comments, impressions, expectations and common cultures provide the main ground and the main basis for the establishment of trust-oriented communication networks because trust is affected by the social background as it differs in different cultures, nations and companies.

This is the trust which create social capital among individuals therefore it is considered as the 
necessary element of the relations among actors. Trust presents three important functions as follows:

-The structure for obligations, expectations and the quality of trust

- Communication and information channels

-A system of norms and effective sanctions (Chining Lung, 2004)

In short the social capital provides information and control advantages through establishment of relationships between individuals who are probably separated from the social structure. Meanwhile certain types of the social structures are playing important role in facilitating certain forms of the social capital (Clement, 1998).

As it was already mentioned we can admit that the human elements within an organization and the interaction and relationships among them are considered as the major elements in the organizational knowledge management (Sivi, 2000, Goicher, 2000). For implementation of the knowledge management processes interaction and relationships between individuals and within the organization types of human relationships are of paramount importance. Any organization which can promote effective interaction among its staff and within different organizational groupings and departments can accordingly ensure creation of new knowledge within the organization and further transfer and exchange of knowledge among the staff as well as the effective knowledge management (Baker. 2000). Hence, creation and development of the culture and atmosphere within the organization which facilitates such relationships and interaction is among the necessities of the knowledge management relevant activities.

As it was already said the most important element in the social capital is the networks of communication which are considered as valuable assets for every organization and its staff. The social capital is considered as a privilege that people within the social networks can enjoy due to the existence of common cultural norms, effective interaction, mutual trust and personal relations among themselves.

As the definitions already refereed to are implying and as many researchers has been emphasizing the more an organization enjoys human capital the more atmosphere of mutual trust-oriented human interaction and collaboration can be witnessed within that organization. Therefore given the concept of the social capital and the need for further promotion of the knowledge management different researchers and scholars have already reached the conclusion that they need to design and test relationship between the social capital and different dimensions, processes and activities of the knowledge management within an organization. In other words the more an organization enjoys social capital the more the basic knowledge management activities (knowledge acquirement, registration, saving, transfer, creation and utilization) will be facilitated and promoted within the organization. As a matter of fact development of the social capital is considered as one of the pressing requirement of any learning and knowledge organization and the knowledge management itself (Adler and Kwon, 2002; Huffman, 2005).

So far a number of experimental studies have been carried out by different researchers on the relationship between the social capital and different knowledge management related activities. Based on their research in 2002 Adler and Kwon reached the conclusion that there exists a meaningful relationship between the social capital and knowledge transfer. Within the same 
respect Hunt (1999) obtained fruitful evidences on the existence of relationship between the social capital and the knowledge management. Kogut (2002) also acquired sufficient evidences for the mutual effect of the social capital and its creation and utilization.

In his research in 1999 Cohen laid emphasis that there is meaningful relationship between the organizational social capital and the knowledge management. Hoffman, in his research in 2005 also displayed the existence of a relationship between the social capital and the knowledge management and accordingly higher performance of the organization.

On the factors affecting the knowledge management outstanding studies have already been carried out and different factors affecting the knowledge management have been investigated and analyzed. This occurs while on the impact of the social capital upon the knowledge management very limited number of studies been done most of which by going through the relevant literature just attempted to presume the existence of a reasonable relationship between the social capital and the knowledge management. Therefore in the present research the researcher intended to study the impact of the social capital on the effectiveness of the knowledge management and its related activities and carried out an experimental test in this respect with the purpose of clarifying the role of the social capital in the promotion of development and effectiveness of the knowledge management within the Iranian organizations.

\section{Analytical model of the research:}

By going through literature and theoretical foundations of the present research dimensions of the social capital based on the Nahapiet and Ghoshal (1998) view point was picked for investigating the relationship between the social capital and the effectiveness of the knowledge management. In the analytical model of the research the social capital was used as the independent variable and the knowledge management was used as the dependant variable. The social capital includes three (cognitive, relational and structural) dimensions and the knowledge management comprises of four dimensions of knowledge creation, knowledge organization, and knowledge sharing /distribution and knowledge application/utilization.

\subsection{Primary assumption:}

-There is a meaningful relationship between the social capital and knowledge management effectiveness

\subsection{Secondary assumptions:}

1- There is a meaningful relationship between the structural dimension of the social capital and effectiveness of the knowledge management

2- There is a meaningful relationship between the relational dimension of the social capital and effectiveness of the knowledge management

3- There is a meaningful relationship between the cognitive dimension of the social capital and effectiveness of the knowledge management 


\section{Research methodology:}

In view of the objectives of the present research it was considered as an applied research. The design of the present research was descriptive (survey); descriptive because it provided us with an image of the present status and surveying because all the information was collected through distribution of the questionnaires distributed among the staff of the Iran Khodro Diesel Company Research and Development Department.

For calculation of volume of the required sampling the following formula was used:

$$
n=\frac{N \times Z \alpha^{2} / 2 \times P(1-P)}{\varepsilon^{2}(N-1)+Z \alpha^{2} / 2 \times P(1-P)}
$$

; Wherein value of $\mathrm{P}$ equals 0.5.; because if $\mathrm{P}$ equals 0.5, " $\mathrm{n}$ " would have its maximum value. This made have the sample big enough (Azar Momeni, 2001) for the purpose of the research. Therefore volume required by the research was calculated as follows:

$$
\frac{152 \times 1.96 \times 1.96 \times 0.5 \times 0.5}{151 \times 0.04 \times 0.04+1.96 \times 1.96 \times 0.5 \times 0.5}=121.44 \sim 121
$$

For designing the questionnaires of the research, theoretical foundations, the outcome of some studies already carried out in this respect and views of the relevant experts as well as guide and advisor professors' views were applied.

For assessment and measurement of the responses provided by the responders for the present research the Five-Point Likert was used (from too much option to too little option).

For calculation of " $\alpha$ " coefficient the SPSS software was used. The value of " $\alpha$ " in the knowledge management questionnaire calculated at 0.89 and the value of " $\alpha$ " coefficient in the social capital questionnaire was calculated at 0.91 all implying validity of the questionnaires. But because " $\alpha$ " coefficient was verified more than the acceptable limit for the applied purposes (more than 70\%) it was admitted that the said questionnaire was of acceptable reliability. For validity measurement the structural equations were utilized and the factor analysis also used for the reliability measurement. The factor analysis of the research model and the research questionnaires was carried out as follows:

Chart No.1 demonstrates the first model for measuring the social capital at the standard estimation mode. Results of the estimation (lower part of the chart) are implying goodness of fit of the model. Given the LISREL output, value of $\mathrm{X}^{2}$ calculated at 93.43 which considered as a low but acceptable value. Low value of this indicator implied that there was very little difference between the research conceptual model and the data of the research. Value of the RMSEA was calculated at 0.000 . The closer this figure is to zero fitness of the model is more. And because the model was of good fitness and the value of RMSEA was equal zero other indicators like AGFI and GFI were no used. 


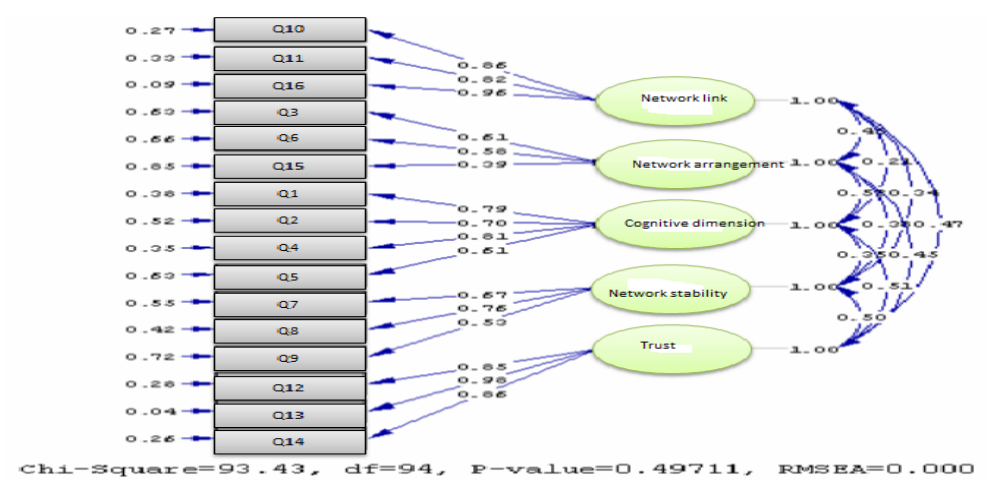

figure1. Social capital measurement model at standard estimation mode by use of the factor analysis

Or the second order factor analysis of the social capital the second measurement model for the social capital was utilized. Again the outcome was implying goodness of fit of the measurement model (Chart No.2).

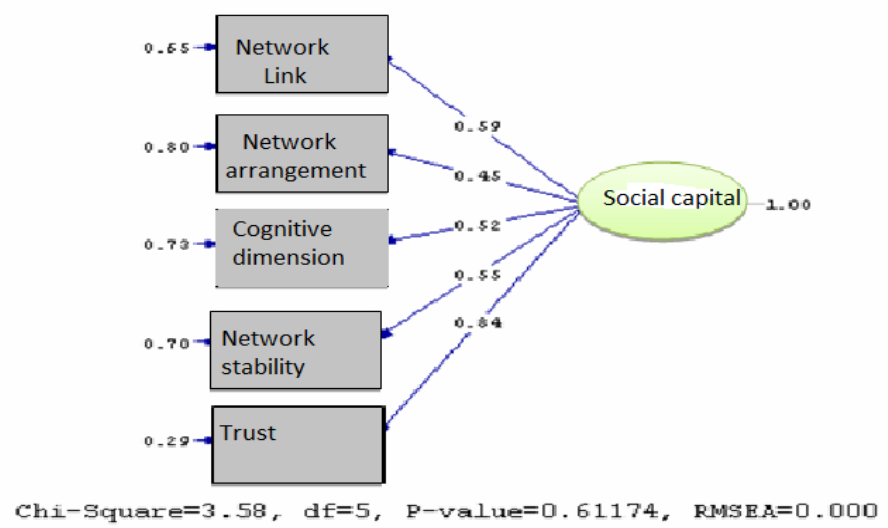

Figure2. Second social capital measurement model and the factor analysis at standard estimation mode

The results of the model at standard estimation mode indicated that out of the identified five dimensions in the social capital trust was of the most impact upon the social capital. Trust was of the highest denotative standard equaling to 0.84 out of other factors and dimensions affecting the social capital. Based on the findings we can admit the significance of the relational dimension and trust among the staff within an organization for creation of the social capital.

Therefore the results of use of the first and the second measurement models for the social capital was indicative of the goodness of fit of the conceptual model of the present research. 
Value of RMSEA and $\mathrm{X}^{2}$ and fitness of these models were indicative of conformity of the conceptual model with the available data. After use of the measurement model of the social capital and confirmatory factor analysis in this part, results of the use of the measurement models of the knowledge management and the confirmatory factor analysis for this variable were presented by use of the path analysis and the structural equation models through application of the LISREL software. The results were implying the goodness of fit of the research model and meaningfulness of the first-order factor analysis of the knowledge management data. Chart No.3 is displaying the first measurement model of the knowledge management along with the adjusted goodness of fit indexes. Indexes of the model are as follows:

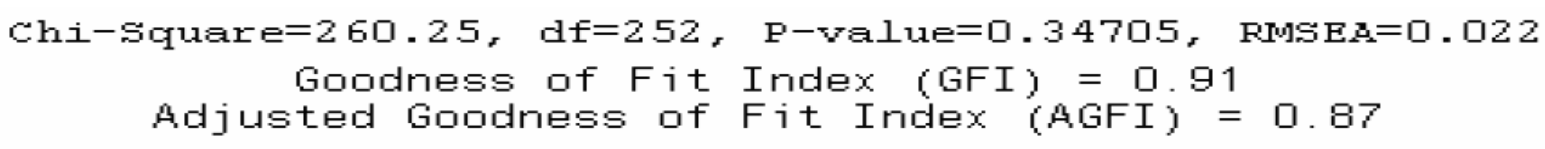

As it has been displayed the model indexes were indicative of the model goodness of fit. Value of $X^{2}$ was low and good (if $X^{2}$ / df stands less than 3 then the $X^{2}$ value will be low and acceptable therefore the model will be of relative goodness of fit. In other words the lower the $\mathrm{X}^{2}$ values (in other words X2 / df) better fitness the model enjoys. In the present model the ratio was about 1). Also RMSEA value was calculated at less than 0.05 and GFI (0.91) and AGFI (0.87) indexes were implying fitness of the model. This means that the conceptual model of the present research enjoys high rate of conformity with the relevant data. 


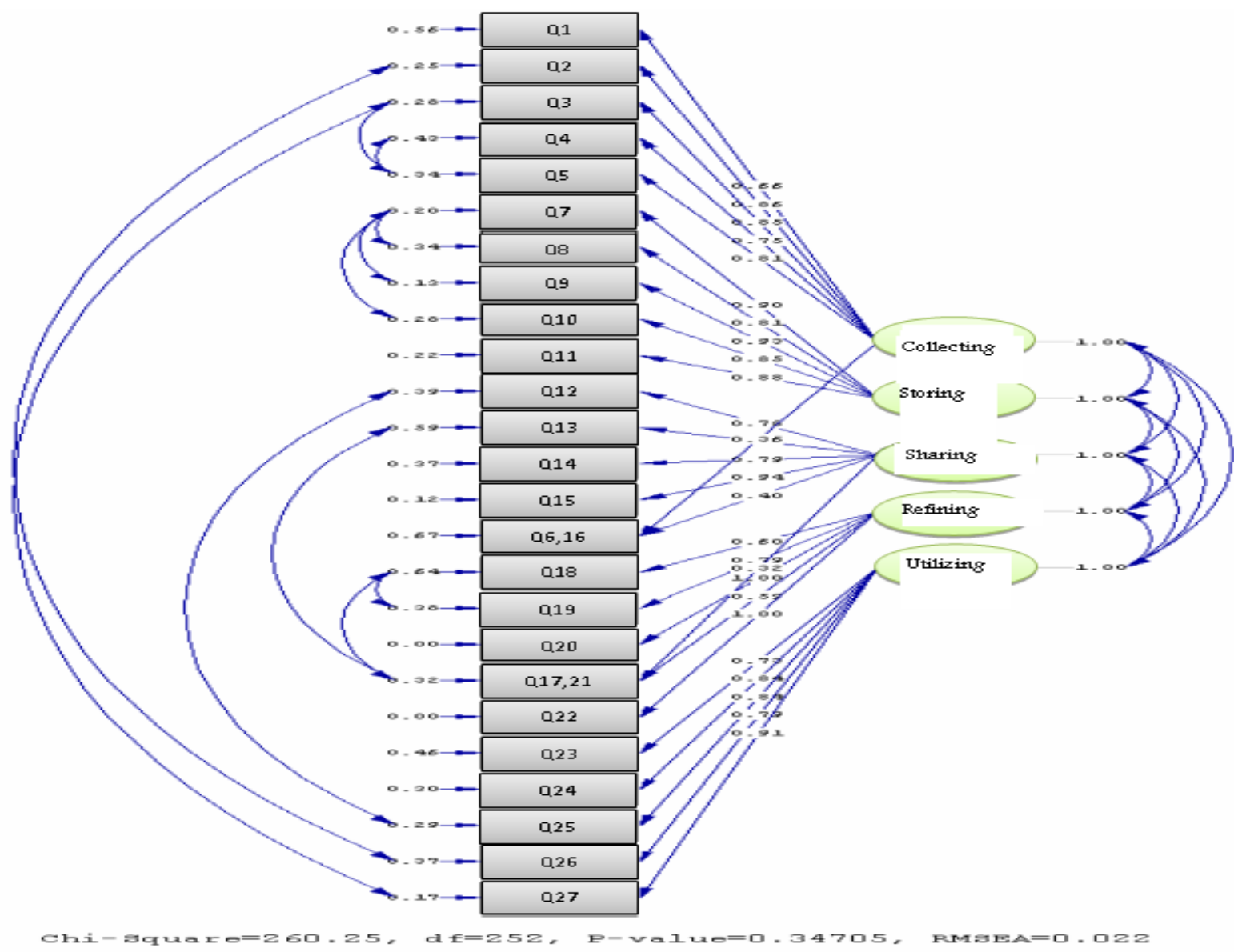

Figure3.Measurment Model of the knowledge management based on the factor analysis with standard estimation mode

In conformity with the second-order factor analysis for the knowledge management, the second measurement model of the knowledge management was used. The software output has been demonstrated in the figure 4 . 


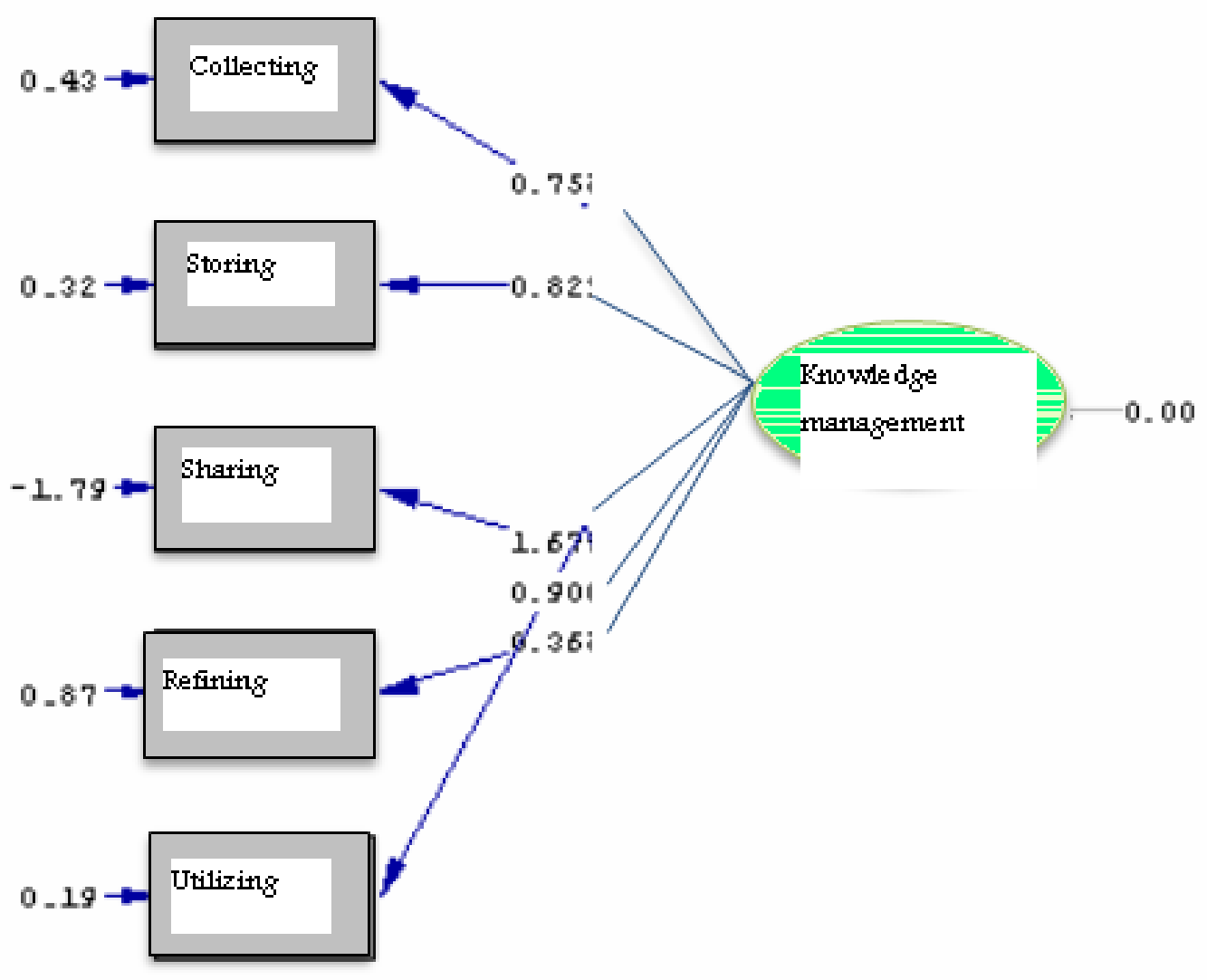

Chi-Square=3.06, df=5, P-value=0.69125, RMSEA $=0.000$

Figure 4.The software output

The results of use of the model were indicative of fitness of the conceptual research model and meaningfulness of the second-order factor analysis of the knowledge management data. The above chart is displaying the knowledge management model along with the adjusted goodness of fit indexes. The indexes of the model were as follows:

As it has been displayed the model indexes were indicative of the model goodness of fit. Value of $\mathrm{X}^{2} / \mathrm{df}$ was equal to 1 . So the $\mathrm{X}^{2}$ value was assesed low and acceptable. Other indexes were implying goodness of fit of the model. Also RMSEA value was equal to 0.000 and less than 0.05. GFI (0.98) and AGFI (0.95) indexes were implying the model goodness of fit. This means that the conceptual model of the present research enjoyed high rate of conformity with the relevant data.

Based on different analysis already referred to in the previous pages the primary of the present research was confirmed as follows: 


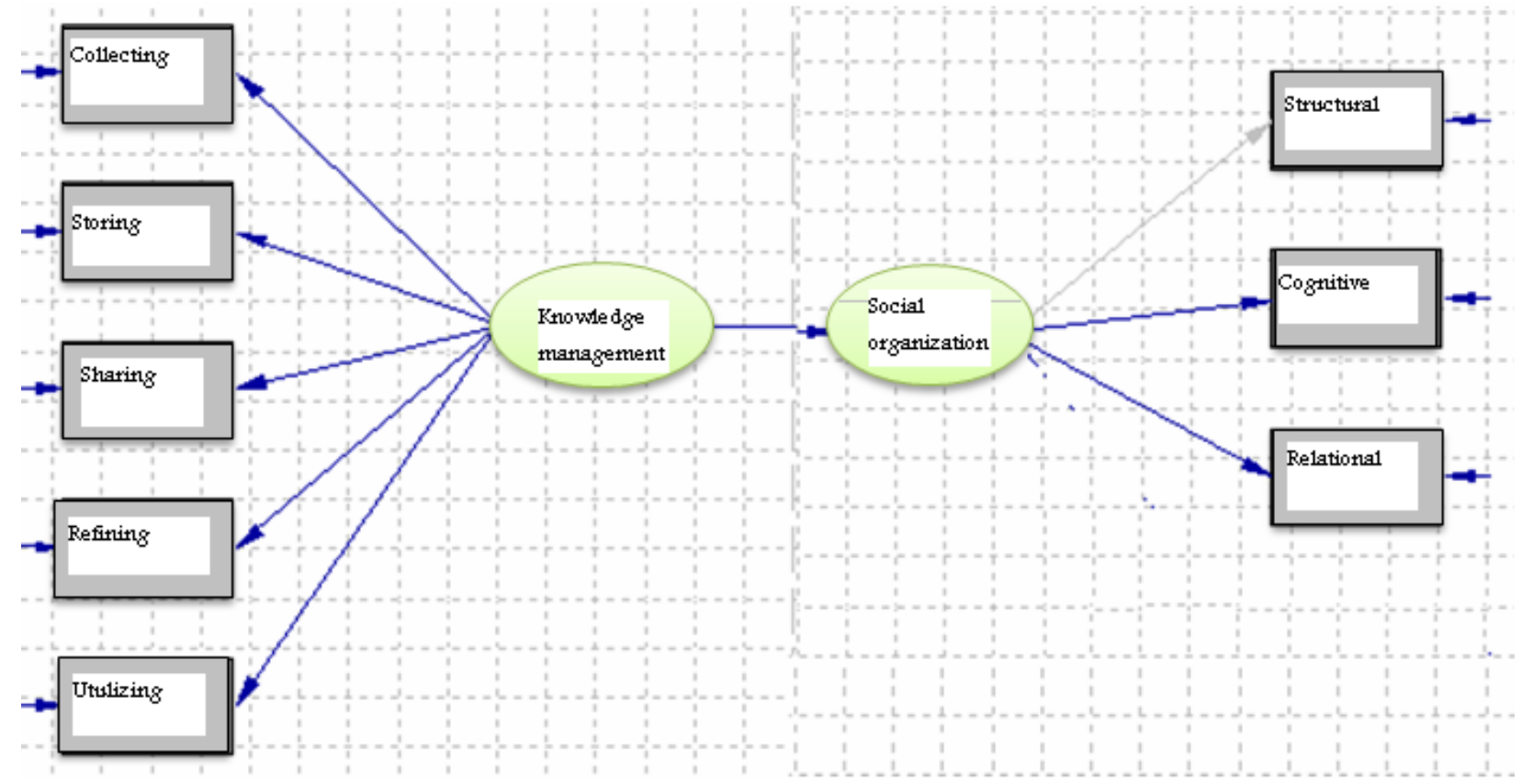

\section{Inferential statistics}

For testing normality of the data collected for the knowledge management model the Kolmogorov-Smirnov test (K-S test) was used.

Table1. the normality test: For variable of knowledge management and its indexes

\begin{tabular}{|c|c|c|c|c|}
\hline Variable & $\begin{array}{c}\text { Meaningfulness } \\
\text { level }\end{array}$ & Error rate & $\begin{array}{c}\text { Confirmation of } \\
\text { the assumption }\end{array}$ & Conclusion \\
\hline Collecting data & 0.01 & 0.05 & $\mathrm{H} 1$ & Not normal \\
\hline $\begin{array}{c}\text { Knowledge storage } \\
\text { and organization }\end{array}$ & 0.01 & 0.05 & $\mathrm{H} 1$ & Not normal \\
\hline Knowledge sharing & 0.014 & 0.05 & $\mathrm{H} 1$ & Not normal \\
\hline $\begin{array}{c}\text { Continuous } \\
\text { knowledge refining }\end{array}$ & 0.021 & 0.05 & $\mathrm{H} 1$ & Not normal \\
\hline $\begin{array}{c}\text { Knowledge } \\
\text { utilization }\end{array}$ & 0.038 & 0.05 & $\mathrm{H} 1$ & Not normal \\
\hline
\end{tabular}

Because the meaningfulness of all indexes was less that the error rate of 0.05 then we concluded that the knowledge management variable and its indexes did not follow normal sharing of knowledge. 
Table 2. The normality test: For variable of the social capital and its indexes

\begin{tabular}{|c|c|c|c|c|}
\hline Variable & $\begin{array}{c}\text { Meaningfulness } \\
\text { level }\end{array}$ & Error rate & $\begin{array}{c}\text { Confirmation of } \\
\text { the assumption }\end{array}$ & Conclusion \\
\hline $\begin{array}{c}\text { Cognitive } \\
\text { dimension }\end{array}$ & 0.001 & 0.05 & $\mathrm{H} 1$ & Not normal \\
\hline $\begin{array}{c}\text { Structural } \\
\text { dimension }\end{array}$ & 0.002 & 0.05 & $\mathrm{H} 1$ & Not normal \\
\hline $\begin{array}{c}\text { Relational } \\
\text { dimension }\end{array}$ & 0.002 & 0.05 & $\mathrm{H} 1$ & Not normal \\
\hline
\end{tabular}

Because the meaningfulness of all indexes was less that the error rate of 0.05 then we concluded that the social capital variable and its indexes did not follow normal sharing of knowledge.

Because based on the data collected from the questionnaires none of the dimensions found normal for further examination of the discussed dimensions the Spearman's rank correlation coefficient was used.

\section{Test on the First Assumption:}

There is a meaningful relationship between the structural dimension of the social capital and effectiveness of the knowledge management

In order to confirm or reject the assumption the available data relating to the structural dimension of the social capital and the knowledge management effectiveness collected through the questionnaires were analyzed by use of Spss and Kolmogorov-Smirnov test (K-S test). In the end it was verified that between the structural dimension of the social capital at the meaningfulness of 0.021 with correlation efficient of 0.602 and the knowledge management effectiveness there is a meaningful and direct relationship. Therefore it was concluded that the more the organization moves towards development and promotion of the structural dimension of the social capital the more the effectiveness of the knowledge management will be promoted and facilitated.

\section{Test on the Second Assumption:}

There is a meaningful relationship between the relational dimension of the social capital and effectiveness of the knowledge management

In order to confirm or reject the assumption the available data relating to the relational dimension of the social capital and the knowledge management effectiveness collected through the questionnaires were analyzed by use of Spss and Kolmogorov-Smirnov test (K-S test). In the end it was verified that between the relational dimension of the social capital at the meaningfulness of 0.000 with correlation efficient of 0.522 and the knowledge management effectiveness there is a meaningful and direct relationship. Therefore it was concluded that the more the organization moves towards development and promotion of the 
relational dimension of the social capital the more the effectiveness of the knowledge management will be promoted and facilitated.

\section{Test on the Third Assumption:}

There is a meaningful relationship between the cognitive dimension of the social capital and effectiveness of the knowledge management

In order to confirm or reject the assumption the available data relating to the cognitive dimension of the social capital and the knowledge management effectiveness collected through the questionnaires were analyzed by use of Spss and Kolmogorov-Smirnov test (K-S test). In the end it was verified that between the cognitive dimension of the social capital at the meaningfulness of 0.014 with correlation efficient of 0.672 and the knowledge management effectiveness there is a meaningful and direct relationship. Therefore it was concluded that the more the organization moves towards development and promotion of the cognitive dimension of the social capital the more the effectiveness of the knowledge management will be promoted and facilitated.

\section{Primary Assumption:}

There is a meaningful relationship between the social capital and knowledge management effectiveness

The primary assumption was related to the existence of a meaningful relationship between the social capital and knowledge management effectiveness within the Iran Khodro Diesel Company Research and Department. In order to test the assumption the Spearman's rank correlation coefficient between the social capital and the knowledge management within the Iran Khodro Diesel Company Research and Development Department was calculated at 0.512 which indicated that the relationship between the said two variables is meaningful and the social capital in the company is of basic role in materialization of the knowledge management in the company and the more the social capital gets strengths the more success will be followed by establishment of the knowledge management. The reference made in the assumption is suggesting that by increase or decrease of the numerical value of the social capital components of the knowledge management will increase or decrease accordingly. Based on the findings of the present research, outcome of the studies made by Alderon (2002) on the relationship between the social capital and the knowledge transfer and Kugat (2004) on the relationship and mutual effect of the social capital with creation and application of knowledge was confirmed. The results of research also were found in conformity with findings of the studies carried out by Haunt (1999), Cohen (1999) and Hofmann (2005) suggesting that there is relationship between the social capital and the knowledge management. 
Table 3: Average of the ranks in the Friedman test with the purpose of investigating factors relating to the knowledge management

\begin{tabular}{|c|c|}
\hline $\begin{array}{c}\text { Factors relating to the knowledge } \\
\text { management }\end{array}$ & Average rank \\
\hline Knowledge collection & 3.57 \\
\hline Knowledge storage and organization & 4.16 \\
\hline Knowledge sharing/distribution & 2.30 \\
\hline Continuous knowledge refinement & 2.54 \\
\hline Knowledge utilization & 2.44 \\
\hline
\end{tabular}

Based on the Table 3 knowledge storage and organization holds the first rank and knowledge sharing holds the fifth rank. As we can see in the Table5.2 the highest rank goes to the relational dimension and the lowest rank goes to the structural dimension of the social capital.

Table 4: Average of ranks in the Friedman test with the purpose of investigating factors relating to the social capital

\begin{tabular}{|c|c|}
\hline Factors relating to social capital & Average rank \\
\hline Cognitive dimension & 3.72 \\
\hline Structural dimension & 3.31 \\
\hline Relational dimension & 3.95 \\
\hline
\end{tabular}

\section{The comparison between findings of the present research and findings of the previous} studies

In the research carried out in the Daadepardazane Iran Company the same subject had been investigated. During the research the research titled "Role of the social capital in development of the organizational knowledge management" the following assumptions were raised and discussed by the researcher:

1- The Social capital is affecting the hard measures of the knowledge management

2- The Social capital is affecting the soft measures of the knowledge management

Based on the research outcome the first assumption was confirmed and the second one was rejected. It is noticeable that since the activities of knowledge acquirement, knowledge registration and knowledge application were identified as hard activities no relation was confirmed in this respect and knowledge soft activities including knowledge creation and knowledge transfer was found having meaningful relationship with the social capital. It is worth mentioning that soft activities of the knowledge management include those activities in the knowledge management cycle that are mostly based on the human factor, human interaction and effective human communications. The hard activities of the knowledge management also include structure and technology oriented activities with in the organization.

The outcome of Nemati and Jamshidi research titled "Relationship and Impact of Knowledge Sharing Process on the Social Capital among Members of the Technologies Units of the Roshd Center of Shaid Beheshti University Branch in Mashhad" also confirmed the third 
assumption (there is meaningful relationship between the knowledge exchange process and experience).

Role of the social capital in knowledge sharing/dotribution and creation also was confirmed in the research carried out by Moshabaki and Qelichkhani (on two Iran's automobile manufacturing companies). At the end of their research Moshabaki and Qelichkhani concluded that:" The social capital as one of the most important organizational features can assist the desired company in creating and sharing knowledge and in promotion of its intellectual asset leading to establishment of the sustainable organizational advantage".

\section{Recommendations for development of the knowledge management:}

Development of the knowledge management related activities requires provision of desirable and necessary conditions and pre-requisites. Structure, technology, culture, communications and strategies all are needed to take form for development and promotion of the organizational knowledge management and required reconsidered and redesigned. Based on the outcome of the present research if any organization wishes to materialize desired knowledge management and organizational knowledge management it must provide an atmosphere within the organization filled with mutual trust and effective communication and interaction among its staff to that extent the said atmosphere give way to promotion of exchange of views and ideas in favor of further renovation within the organization. Provision of suitable grounds in the matter of structure and technologies is of vital significance in developing the knowledge management. In another word if the knowledge which is created within the organization and subsequently transferred is not documented and registered within the organization and it can not be utilized in a desirable manner and it will not positively affect the organizational competitiveness or it will be followed by just very limited positive output.

\subsection{Some recommendations for improvement of the knowledge collection or acquirement:}

Cooperation between organizations and companies on one side and universities and research institutes on the other side in order to identify future opportunities.

Active involvement of the staff of all organizations and companies in the scientific, specialized and professional networks.

Active involvement of the all organizations and their staff in the training and vocational courses.

Cooperation with rival companies and organizations as valuable sources of information.

\subsection{Recommendations for improvement of knowledge and information storage:}

Purchase of books and up-to-date information sources in order to be used by staff

Assessment of achievements and failures and their documentation and registration

Registration and documentation of knowledge and skill of experts and managers of the 
organization

\subsection{Recommendations for promotion of knowledge creation}

To organize meetings and seminars as well as brainstorm sessions for identifying new resolutions and discussing specialized subjects.

To welcome new ideas and knowledge and create innovation-oriented culture within the organization.

To reward innovations and initiatives.

\subsection{Recommendations for promotion of continuous knowledge refinement}

Organization of meetings and seminars for proper knowledge transfer and exchange of expertise among the staff.

Provision of suitable atmosphere and culture of mutual trust for promotion of the transfer and exchange of knowledge among the staff.

Periodical relocation and appointment of the staff to new posts and different departments.

\subsection{Recommendations for development of knowledge and information utilization}

Precise assessment and utilization of the ideas and suggestions submitted by the staff Precise assessment of the available knowledge in industry and the knowledge possessed by rival companies and organizations and making every effort to acquire and apply it Implementation different types of researches in the fields of research and development and the market for utilizing new knowledge within the organization Provision of required infrastructures and technologies like information technology, software groups and databases for promoting the knowledge management

\subsection{Recommendations for development of the social capital}

Given the fact that findings of the present research are implying that development and promotion of the organizational social capital are affecting the organizational knowledge management especially the soft measures of the knowledge management (knowledge creation and transfer) the following measures can be adopted in favor of the social capital promotion:

1- Vertical and horizontal relationships shall be promoted. The vertical and horizontal relationships relates to the modality of decision making process within networks or essence of the execution of law. Within the same respect Chrishna and Sherader are referring to the essence of the power relations within networks and the decision making processes. For materialization of the said objective the following mechanisms can be utilized.

Handover of more authority to the lower ranks within the organization by senior managers and directors 
Use flatter organizational structure that enjoys a structure with less complexity, formality and centrality

Use of informational systems

2-The second way or method in promoting the social capital within the organization is to enforce trust. The following measures may be adopted for enforcement of trust:

The norm of trust is considered as the insuperable part of the social capital. The norm of trust is formed in the course of social relations and prepares the ground for further interaction and cooperation among the staff of the organization. Transfer of information and knowledge, whether at macro and micro levels, among individuals and organizations depends upon people who facilitate and begin the transfer. Consequently all things which impede or persuade relationships among individuals will affect the knowledge transfer. For the same reason trust has always been given significant priority. Trust becomes more important particularly when the relationships are complex. Therefore trust is of utmost importance in knowledge sharing. In other words, when some risks and uncertainty are visible in the knowledge exchange we can settle the uncertainty by use of high level of trust which by itself is of great importance for the effective function of the knowledge management. So, for promoting the trust we need to bring the following into our attention:

Adherence to moralities: Those managers who give significance to the moral values in the organizational performance and decisions in fact are acting as elements who are marching towards further promotion of trust within their organizations or companies.

Sense of responsibility: whenever staff of an organization rest assured that their managers feel themselves responsible to their staff and accountable to the society then their positive feelings to their organization will promote. In the light of such a promotion the trust supersedes the distrust within the organization.

To make the staff further involved in the higher levels of organization and to make them familiarized with the problems, weak points and strong points of the organization will lead to further trust within the organization.

3-Promotion of mutual bindings among the staff within an organization will further facilitate exchange in the social relations leading to further exchange of services and commodities. The mutual relations are subject to a set of norms which themselves are subject to different types of networks. In order to promote bindings among networks we can take the following measures:

To persuade the moral for assisting other employees among the staff within the organization using the managers as the symbol

To reward such positive behaviors

\section{References:}

1- Manuriyan Abbas, 2006, "knowledge management”, work and society, P.73-74.

2- Mac Donald John, translated and collected by: Iran Expert Group, 2002, "knowledge management in a single week, Quality and Management.

3- Alavi Seyyed Babak, 2001, "Role of the Social Capital in Development", Monthly periodical of "Tadbir", No. 116. 
4- Damuri Daryush, Mansuri Hossein, Taheri Mohsen, 2009, "Study of Relationship between Knowledge Management and The Social Capital In the Islamic Azad University", quarterly of "Management", Azad University, $13^{\text {th }}$ year of publication, No. 1 spring of 2009.

5- Alvani Seyyed Mehdi and Mir Ali Seyyed Naqavi, 2002, "The Social Capital: Concepts and Theories", quarterly of "The Management Studies”, No. 33-34, P.3-26.

6- Woolcock, M., \& Narayan, D., (2000), "Social Capital: Implications for development theory, research, and policy", World Bank Research Observer, Vol. 15, NO. 2, pp. 2_27.

7- Nahapiet, J. , \& Ghoshal, S., (1998), "Social Capital, Intellectual Capital \& the organizational advantage", Academy of management Review, Vol. 23, NO. 2, pp. 242_260.

8- Lock Lee, Laurence, (2005), "Schemes and Tools for Social Capital Measurement as a proxy for Intellectual Capital Measures".

9- Leana, C.R \& H.J. Van Buren, (1999), "Organizational Social capital and Employment Practices", Academy of Management Review, Vol. 24, No.3, pp. 538_555.

10- Islam M. K, \& J. Merlo, \& I. Kawachi, \& M. Lindstrom, \& U.G, Gerdtham (2006), "Social capital and health: Does egalitarianism matter? A Literature Review", International Journal for Equity in Health, Vol. 5, No.3.

11-Francis, P., (2002), "Social Capital at World Bank: strategic and operational Implications of the concept", social development strategy, World Bank.

12- Carrie, R. Leana, Frits K. Pil ,(2006), "Social Capital and Organization Performance: Evidence from Urban Public Schools", Organization Science,Vol.17, No.3, pp.353_366.

13-Wong, K. Y., \& Aspinwall E. (2010), Development of a knowledge management initiative and system: A case study. Journal of Expert system of application, 30(3), 633-64 1.

14-watson jan , (2003)," Applying knowledge management : techniques for building corporate memories" morgan kaufman publishers,pp. 4,5,7

15-Timberlake, Sharon, (2005), "Social capital and gender in work place", Journal of Management Development, Vol. 24, NO 1.

16- steven Walczak.(2005),"organizational knowledge management structure".journal of the learning organization ,vo1,12,no4 , pp . 330-337

17- Ramanujan, S. and Someswar, K., (2010), "Comparison of knowledge management and CMM/CMMI implementation", Journal of American Academy of Business, 4(1-2), pp. 271-275. 
18- Potts, Helen, B.S., (2007), "The Role of social capital in organization: The precursors and effects of social capital among certified nurse aides in nursing homes", University of North Texas, pp. 93_95.

19-nonaka , I . ( 2005 ) ." Dynamic theory of organizational knowledge creation ", ln : organization science, vol , 5 No . $1: 14-35$

20-Leitner, K.H. \& C. Warden (2010). Managing and Reporting knowledge based Resources

21-johnson David, (2005 ) ", innovation and knowledge management : cancer information service research consortimum" . publisher by Edward elgar publishing limited .

22- Hodson, R., (2005), "Management behavior as social capital: a systematic analysis of organizational ethnographies", British Journal of Industrial Relations, Vol. 43, No. 1, pp. 41_65.

23-Francis, P., (2009), "Social Capital at world bank: strategic and operational Implications of the concept", social development strategy, world bank.

24-Desouza, k.c, Awazu,y.(2006)" Engaging tensions of knowledge management control".singapour management review.

25-Coleman, J., (2009), "Social Capital in the creation of human capital", American Journal of Sociology, Vol. 94, pp.95_120.

26- Carrie, R. Leana, Frits K. Pil ,(2006), "Social Capital and Organization Performance: Evidence from Urban Public Schools", Organization Science,Vol.17, No.3, pp.353_366.

27-Berman, S., (2007), "Civil Society and Political Institutionalization", American Behavioral Scientist, 40(5).--End 